\title{
The Fascination of Cytokine Immunological Science
}

\author{
Paolo Lissoni'*, Giusy Messina' , Francesco Pelizzoni ${ }^{2}$, Franco Rovelli', Fernando Brivio', Alejandra Monzon' , Na- \\ dal Crivelli', Arianna Lissoni', Simonetta Tassoni ${ }^{3}$, Andrea Sassola', Sonja Pensato', Giuseppe Di Fede' \\ 'Institute of Biological Medicine, Milan, Italy \\ ${ }^{2}$ Cardiological Surgery Division, Niguarda Hospital, Milan, Italy \\ ${ }^{3}$ Effata Institute, Lucca, Italy
}

Article Info

\section{Article Notes}

Received: April 1, 2020

Accepted: April 21, 2020

\section{${ }^{*}$ Correspondence:}

Paolo Lissoni, Institute of Biological Medicine, Milan, Italy; Email: paolo.lissoni@gmx.com.

(c) 2020 Lissoni P. This article is distributed under the terms of the Creative Commons Attribution 4.0 International License.

\section{Keywords}

Cytokines

Cytokine network

Inflammation

Interleukins

\section{Abstract}

Today, it is known that all human biological functions are under two fundamental regulatory systems, consisting of the endocrine system and the cytokine network. Moreover, it has been shown that the cytokines released from the activated immune cells do not influence only immune functions, but also the whole biological system, including the various metabolic activities, the cardiovascular system, and the functionless of the neuroendocrine system itself. Unfortunately, despite the well-demonstrated importance of cytokines in maintaining the status of health, from a clinical point of view the routine evaluation of the cytokine system still remains unconsidered to establish the status of health, since it is investigated only in severe conditions, such as septic shock, disseminated intravascular coagulation and respiratory distress, which have been demonstrated to be due to an abnormal endogenous production of inflammatory cytokines, namely IL-6, TNF-alpha, and IL-1 beta. This clinical deficiency was depended on several factors, particularly on the complexity of cytokine interactions themselves, but also on the decision to use artificial molecules, such as monoclonal antibodies against the various cytokines, to counteract their eventual abnormally enhanced endogenous production, rather than to investigate the mechanisms responsible for their altered production and to correct eventual alterations. The main reason of the complexity of the cytokine network is related to the fact that the interactions occurring among the different cytokines are often founded on positive feedback mechanisms, then on reciprocal stimulatory actions, while the endocrine system is substantially based on negative feedback circuits. The aim of the present review is to propose a synthetic knowledge regarding the main effects and the source of origin of each single interleukin discovered up to now, to elaborate a first preliminary fundamental physiology of the cytokine network.

\section{Introduction}

As "cytokines", we define a great group of small proteins produced by the immune cells after their activation, as well as by stromal cells, involved in the modulation of the biological immune-inflammatory response, and which are responsible for the pathogenesis of human systemic diseases. The cytokines more exactly produced by lymphocytes were defined with the term of lymphokines. Finally, cytokines received their interleukin (IL) definition at the second International Lymphokine Conference in Interlaken (Switzerland) in 1979. Since then, the number of interleukins has grown significantly. At the end of $21^{\text {st }}$ century, the total group of interleukins has reached the number of 30, and kept on increasing more, and at present there are currently 40 interleukins named. Obviously, there are only few studies concerning the biological significance of the last discovered 10 interleukins. Moreover, since the last discovered interleukin in 
its complete structure was IL-40 in $2017^{1}$, and no other interleukin was discovered during the last two years, we may consider, at least from a synthetic and symbolic point of view, that the number of interleukins may be 40 . Finally, some interleukins have received a great attention by the Immunologists, while others have been less investigated, since they were considered to play a less importance role in the human biology. In any case, the classification of interleukins themselves is still ambiguous, since there is no clear definition of interleukins with respect to that of cytokines. Then, some cytokines provided by important biological effects are not defined as interleukins, for example tumor necrosis factor-alpha (TNF-alpha) and transforming growth factor-beta (TGF-beta), which are respectively characterized by proinflammatory, or by antiinflammatory effects. Today, it is known that the human systemic diseases may be interpreted as the end-result of an interaction between the action of some exogenous agents and host biological response. Therefore, the human pathologies could be sub-divided into two major classes, consisting of diseases namely depending on the action of exogenous agents, including microbes and potential toxic or cancerogenic agents, and diseases mainly due to host immune-biological response. The human systemic diseases, including cancer, autoimmune pathologies and allergy, would depend at least in part on host biological response, consisting of an exaggerated immune reaction, such as autoimmune and allergic pathologies, or a deficient immune response, such as that occurring in the neoplastic diseases. The endocrine mechanisms may explain some human pathologies, but the most severe human diseases would be due to an altered immune function. Moreover, while the endocrine system is regulated by negative feedback mechanisms, which may be understood on the basis of rationale considerations, the biology of the cytokine network cannot be explained only in relation to a logical ratio, since most cytokine secretions are linked by reciprocal stimulatory effects based on positive feedback mechanisms, as well as since some cytokines may induce the secretion of other cytokines provided by opposite activities in an attempt to counteract their excessive effects on the biology of the living organisms. Therefore, we need a different mental approach with respect to the simple mechanic logical ratio, also founded on symbolic archetypic dynamics. In addition, because of the complex interactions among the different interleukins, as well as because the in vivo existence of a physiological neuroendocrine regulation of the cytokine network and interleukin secretion ${ }^{2}$, cytokine effects observed in vitro may be different from those occurring in vivo, and this difference may represent a limiting factor to understand the real impact of the single interleukin in the pathogenesis of human diseases, as well as to establish the possible therapeutic use of each single interleukin in the various pathologies, Therefore, from a therapeutic point of view, in vivo it is more easy to act by blocking an eventual exaggerated secretion of some cytokines by the administration of specific monoclonal antibodies against the single interleukin, whose endogenous production has been proven to be abnormally increased in each single pathology, such as the enhanced production of IL-6, TNF-alpha and TGF-beta in the metastatic cancer ${ }^{3}$ and IL-17 in the autoimmune diseases ${ }^{4}$. On the contrary, in the presence of an interleukin deficiency, such as that of IL-2 in the advanced neoplasms, the therapy will consist of the administration of the interleukin, whose secretion is abnormally low. Finally, the complexity of interleukin system is furtherly amplified by the fact that some interleukins may exist in different isoforms, such as IL-17 in 6 isoforms $s^{6}$, IL-36 in 4 isoforms ${ }^{7}$, and IL-37 in 5 isoforms ${ }^{8}$, as well as by the fact that some isoforms of interleukin may display opposite effects. In vivo, there are two fundamental mechanisms to protect against an eventual excessive secretion of some cytokines, consisting of the production of a soluble receptor, such as for IL-2 ${ }^{5}$, or a binding protein (BP) for a specific interleukin, such as that for IL-18 ${ }^{9}$, which act by reducing its free amount in the blood.

\section{Classification of Interleukins}

The interleukins may be classified according to three main principles, consisting of their family of origin, which is related to their chemical structure, their effects on the inflammatory response, by classifying interleukins in two main groups, respectively provided by proinflammatory or anti-inflammatory activities, and finally their activity on tumor growth. As far as the origin family, it is possible to recognize 7 fundamental family of interleukins, as follows: 1) IL-1 family: this group includes IL-1 beta (since IL-1 alpha is an intracellular protein), IL-18, IL-33, IL-36, IL-37, and IL-38; 2) IL-2 family: it contains IL-2 itself, IL-7, IL-15, and IL-21;3) IL-4 family: it includes IL-4 and IL-13; 4) IL-6 family: it is consisting of IL-6 and IL-31; 5) IL-10 family: it is constituted by several factors, as follows: IL-10, IL-19, IL-20, IL-22, IL-24, IL-26, IL-28, and IL-29; 6) IL-12 family: it contains IL-12, IL-23, IL-27, IL-30, IL-35, and IL-39; 7) IL-17 family: it is characterized by the 6 different isoforms of IL-17 itself. Finally, other interleukins are not classifiable in a specific family of interleukins, including IL-3, IL-5, IL9, IL-8, IL-11, IL-16, IL-25, IL-31, IL-32, IL-34, and IL-40. The IL-12 family is unique in having the only heterodimeric cytokines ${ }^{1}$. On the other side, as far as the relation with their effect on the inflammatory status, interleukins may be subdivided into proinflammatory and anti-inflammatory interleukins. The group of the major proinflammatory interleukins includes IL-1 beta, IL-2, IL-6, IL-8, IL-12,IL-14, IL-15, IL-17, IL-18, IL-20, IL-21, IL-22, IL-23, IL-24, IL-26, IL-25, IL-28, IL-31, IL-32, IL-35, IL-36, and IL-38. On the other side, the group of the anti-inflammatory interleukins includes IL-7, IL-10, IL-19, and IL-38. In any case, it has 
appeared that some interleukins, such IL-2, and IL-12 would be characterized by a dual proinflammatory and anti-inflammatory actions. In more detail, particularly IL-2 and IL-12, which are generally included within the group of the inflammatory cytokines, may play both inflammatory and anti-inflammatory effects. The inflammatory action of IL-2 is mainly due to its stimulatory effect on the macrophage system, whereas the anti-inflammatory one would depend on its inhibitory action on IL-17 secretion and on its promoting effect on TGF-beta secretion ${ }^{5}$. On the other side, the inflammatory action of IL-12 would be linked to its ability to determine $\mathrm{T}$ naïve lymphocyte differentiation into TH1 cells, with a consequent enhanced IL-2 production, and to its inhibitory action on TGF-beta secretion, while its anti-inflammatory effect is mainly depending on the inhibition of IL-17 secretion ${ }^{10}$. IL-3 also may play both inflammatory and anti-inflammatory effect, with the anti-inflammatory activity due to its ability to inhibit IL-2-induced macrophage activation ${ }^{11}$. On the same way, IL-11 may exert both pro- and anti-inflammatory effects, depending on the different experimental conditions, but the main activity would be the anti-inflammatory one $^{12}$. In any case, it is important to remark that there is no relation between interleukin family and its influence on the inflammatory response, since interleukins included within the same family may exert opposite effects on the inflammatory status. Moreover, by considering that the inflammatory status is characterized by interactions between pro- and anti-inflammatory cytokines, it has to be taken into consideration that the classification of interleukins in one or the other category would be too simplistic, since a given cytokine may in some cases exert either pro- or anti-inflammatory effects, depending on the different biological conditions ${ }^{13}$. Inflammatory response itself may determine the release of anti-inflammatory cytokines, such as IL-10, to control and counteract the excessive inflammatory reaction. In addition, it has also to be considered that the anti-inflammatory factors are often represented by soluble cytokine receptors, such as the soluble TNF-alpha receptors p55 and p75, as well as IL-1 receptor antagonist IL-1ra 1 and 2, which reduce the free amount of a given cytokine, rather than by cytokines directly provided by anti-inflammatory effects. In any case, the main confusion is due to the discrepancy between in vitro and in vivo effects of each single cytokine, as shown by the fact that some interleukins generally included within the group of the anti-inflammatory cytokines, such as IL-4 and IL-13, on the basis of their in vitro results, may in vivo present an inflammatory clinical behaviour because of their stimulation of histamine secretion and the possible induction of capillary leak syndrome. The classification of cytokines in relation to their influence on the biological inflammatory response is reported in Table 1 . Another important question regards the actions of the different interleukins on $\mathrm{T}$ lymphocyte proliferation because its influence on the anticancer immunity ${ }^{5}$. Most cytokines tend to exert both anti-tumor and pro-tumor activities, and at present, the only cytokine, which has been proven to really enhance $\mathrm{T}$ lymphocyte count and to determine an evident lymphocytosis in clinical studies still remains IL$2^{5}$, even though IL- $7^{14}, \mathrm{IL}-15^{15}$ and IL- $12^{16}$ could also play a stimulatory action on T lymphocyte proliferation. IL-12 alone induces lymphocytopenia ${ }^{16}$, while the association between IL-12 and IL-2 has been proven to paradoxically determine the maximal lymphocytosis described up to now in the literature ${ }^{17}$. Finally, by considering their action on cancer development and proliferation in clinical conditions, at present the only interleukin clearly provided by anticancer activity in humans still remains the only IL$2^{5}$, and also IL-12, but only in combination with IL- $2^{17}$. In addition, by simultaneously considering the effects on the inflammatory response and on tumor growth, it appears that all clearly inflammatory cytokines tend to display a protumoral action by suppressing the antitumor immunity. Not only, but it has appeared that the main cytokines provided by anti-inflammatory action, namely TGF-beta and IL$10^{18}$, tends to play a pro-tumoral effect by suppressing the antitumor immunity, with the only exception of IL-10, which, in addition to its immunosuppressive activity on both IL-2 and IL-12 secretion, as well as TGF-beta, could potentially stimulate the antigen-dependent cytotoxicity mediated by the cytotoxic $\mathrm{T}$ lymphocytes $(\mathrm{CD} 8+)^{18}$. Then, IL-10 would function as the point of equilibrium between the immune surveillance due to the anticancer immunity, and the suppression of the anticancer immunity. Unfortunately, as observed by Catalan-Dibene et al. ${ }^{1}$, most interleukins were discovered by the older generation of Immunologists, while in contrast the clinical investigation of the importance of cytokines in the pathogenesis of human systemic diseases and their treatment does not seem to be considered as a fundamental topic by the new generation of Immunologists. This evidence could

Table1. Cytokine classification in relation to the effects on the biological inflammatory response.

INFLAMMATORY CYTOKINES

ANTI-INFLAMMATORY CYTOKINES
IL-1 beta, IL-4, IL-5, IL-6, IL-8, IL-9, IL-13, IL-14, IL-15, IL-16, IL-17, IL-18, IL-21, IL-23, IL-25, IL-31, IL-32, IL-33, IL-36, IL-38, IL-39, IL-40, TNF-alpha

IL-7, IL-10, IL-19, IL-20, IL-22, IL-24, IL-26, IL-27, IL-28, IL-29, IL-30, IL-34, IL-35, IL37, TGF-beta

INTERLEUKINS WITH DUAL INFLAMMATORY AND ANTI- IL-2, IL-3, IL-11, IL-12

INFLAMMATORY ACTION 
also be due at least in part to the fact that a complete comprehension of the single interleukin requires several knowledges, including its molecular chemical structure, its receptors, and its biological activity in the different experimental and clinical conditions. Then, from a clinical point of view, it would be enough to synthetically know the main effects of each single interleukin and cytokine, at least on the inflammatory response, lymphocyte proliferation, and tumor cell proliferation, as well as its main source of production.

\section{Main Effects of the Single Interleukin}

\section{IL-1 beta}

IL-1 beta is produced by macrophages. It stimulates the production of IL- 6 from macrophages themselves. Then, IL- 6 is the main cytokine responsible for the induction of the acute inflammatory response by stimulating the hepatic production of acute phase-related proteins, namely the C-reactive protein (CRP) ${ }^{19}$. IL-1 beta would cooperate with IL-18 in inducing macrophage-mediated inflammatory response $^{19}$. IL-1 beta has been proven to induce high levels of serum amyloid $\mathrm{A}$, and to participate in association with IL-18 in the pathogenesis of atherosclerosis ${ }^{19}$. IL-1 beta would represent the main endogenous pyrogenic factor. Moreover, it has been proven to stimulate cortisol secretion during the inflammatory conditions to counteract a possible excessive reaction. Finally, it is also an anorectic cytokine more potent than leptin itself, then it would be responsible for cancer-related loss of body mass ${ }^{19}$.

\section{IL-2}

IL-2 is produced by TH1 lymphocytes, whose differentiation is stimulated by IL-12 released from the mature dendritic cells ${ }^{20}$. IL- 2 is perhaps the main cytokine of the whole immune system, by representing the main cytokine responsible for $\mathrm{T}$ lymphocyte proliferation. In fact, at the beginning of its discovery, it was called as $\mathrm{T}$ cell growth factor (TCGF) ${ }^{13}$. TH1 lymphocytes may secrete IL-2 and concomitantly express IL-2 receptor, with a consequent proliferation of themselves. Then, TH1 would constitute the only immune cells able to realize a selfcloning, by playing an essential role in the functionless of the whole immune system ${ }^{13}$. The fundamental role of IL-2 in maintaining an effective immune functionless is demonstrated by the fact that AIDS-related IL-2 deficiency may allow a complete failure of the immune responses. The antitumor immune action of IL-2 is substantially due to its ability to induce the evolution of NK cells into lymphokine-activated killer (LAK) cells, which are capable of destroying fresh human cancer cells through an antigen-independent cytotoxicity ${ }^{13}$, while NK cells in their basal status are unable to kill fresh human cancer cells, but only laboratory cancer cell lines. The antitumor cytotoxicity played by LAK cells is counteracted by IL- $6^{5}$. Unfortunately, IL-2 may induce a concomitant stimulation of regulatory $\mathrm{T}$ lymphocytes ( $\mathrm{T}$ reg) ${ }^{5}$, with a consequent enhanced production of TGF-beta, which in contrast may inhibit the antitumor immunity, by representing the most active endogenous immunosuppressive agent ${ }^{5}$. In any case, before the recent development of artificial cancer immunotherapies with anti-PD1 or anti-PD-L1 and PDL2, IL-2 therapy had represented the only real scientific immunotherapy of human neoplasms, which would have to be rediscovered and improved on the basis of the successive knowledges concerning the physiopathology of the anticancer immunity. Not only, but IL-2, by stimulating TGF-beta secretion ${ }^{5}$ and by inhibiting that of IL-17 (21), could constitute an effective immunotherapy also for the autoimmune diseases. Then, biological strategies, carried out to modulate TGF-beta response to IL-2, could make IL-2 treatment an effective immunotherapy for both cancer and autoimmune pathologies.

\section{IL-3}

IL-3 is a multi-colony stimulating factor for granulocytes, macrophages, erythroid cells and mast cells, produced by macrophages and stromal cells, and it could be involved in chronic inflammatory conditions ${ }^{23}$. However, IL-3 has also been proven to counteract IL-2-induced macrophage activation, then to exert an anti-inflammatory activity ${ }^{11}$.

\section{IL-4}

IL-4 is a complex cytokine having opposite effects in both cancer and autoimmunity ${ }^{24}$. It is mainly released by $\mathrm{TH} 2$ lymphocytes, and it represents on the same time the main differentiating factor of TH2 cell themselves. At present, it is known that the difference between TH1 and TH2 cells is only functional, since no difference in the expression of clusters of differentiation has been documented between TH1 and TH2 cells. TH1 cells namely release IL- 2 and gamma-interferon (IFN), while TH2 cells may produce IL4, IL-5, IL-6 and IL-10. As far as its possible influence on cancer growth, despite the controversial results reported in the literature, at present IL-4 would seem to play a major pro-tumoral role, because of its stimulatory effect on TGFbeta secretion, and its amplification of IL-17-induced IL-6 production $^{25}$.

\section{IL-5}

IL-5 is the main growth factor for eosinophils. It is released from TH2 lymphocytes under stimulation by IL$2^{5,23}$. In fact, eosinophilia is one of the most typical biological responses to IL-2 administration in cancerimmunotherapy ${ }^{5}$. Eosinophils are mainly involved in the control of protozoan parasites $^{23}$, but it could display a low anticancer activity, at least under IL-2 cancer immunotherapy ${ }^{5}$. IL-3, IL-5, and GM-CSF have been shown to present a common beta chain. 


\section{IL-6}

IL-6 is one of the main inflammatory cytokines, produced by macrophages stimulated by IL-1 beta, as well by TH2 cells. Released under IL- 1 beta stimulation, IL-6 induces the hepatic production of acute phase proteins. IL- 6 exerts a pro-tumoral immunosuppressive activity by counteracting IL-2-induced activation of LAK cells 5 . Moreover, IL-6 would be the main cytokine responsible for septic shock-related untreatable hypotension ${ }^{26}$. IL6 would also exert a partial stimulatory action on platelet generation.

\section{IL-7}

IL-7 is mainly produced by stromal cells, and it has appeared to be required for $\mathrm{T}$ cell development and survival ${ }^{14}$. In more detail, IL-7 would be essential for the transformation of the double negative CD4 and CD8 thymocyte progenitors into double positive CD4 and CD8 thymocytes in the thymus (27). Finally, despite the controversial results, IL-7 would exert a major antiinflammatory and anti-tumor activities ${ }^{27}$.

\section{IL-8}

IL-8 is a like-IFN cytokine, produced by macrophages and stromal cells, with antiviral, inflammatory, angiogenic and pro-tumoral activities. The main target for IL-8 action would be the neutrophils, by stimulating their proliferation and chemotactic activity ${ }^{28}$. High levels of IL-8 have been proven to predict a negative prognosis either in cancer, or in infectious diseases.

\section{IL-9}

IL-9 is a still less investigated cytokine. It is mainly released from TH2 cells, and it would be involved in the pathogenesis of cancer, namely in malignant lymphoma, since high serum levels of IL-9 have appeared to predict a negative prognosis ${ }^{29}$. IL-9 would inhibit the anticancer immunity by stimulating TGF-beta production from $\mathrm{T}$ reg cells, as well as that of IL-17 from TH17 lymphocytes ${ }^{30}$. As far as its effects on the inflammatory status, IL-9 would exert a predominant inflammatory role $^{31}$.

\section{IL-10}

IL-10, namely produced by TH 2 lymphocytes, T reg cells, and macrophages, is one of the main anti- inflammatory and immunosuppressive endogenous factors ${ }^{18}$. It would be also involved in B cell maturation. Despite its inhibitory effect of the anticancer immunity, it could be able of some anti-tumoral activity by stimulating the action of cytotoxic T lymphocytes and their immunological memory ${ }^{18}$.

\section{IL-11}

IL-11 is a multifunctional hematopoietic cytokine, produced mainly by stromal cells under IL-1 stimulation, and it acts in synergistic combination with other cytokines $^{32}$. In addition, it has also appeared to stimulate megakaryocyte proliferation, by acting as a potential growth factor for platelets to increase platelet count ${ }^{32}$. Finally, IL11 has been proven to stimulate the osteoclastogenesis, with a consequent bone loss and osteoporosis ${ }^{12}$.

\section{IL-12}

IL-12, which is produced by dendritic cells and by macrophages when they assume the function of antigen presenting cells, may be considered as the link between innate and acquired immunity. Its main function is the stimulation of the differentiation into TH1 lymphocytes, with the consequent production of IL-2 and gamma-IFN ${ }^{20}$. In association with IL-2, it represents the main anticancer cytokine in humans through several mechanisms ${ }^{5,20}$, including direct activation of antigen-dependent anticancer immunity mediated by cytotoxic T lymphocytes, stimulation of IL-2 secretion from TH1 cells and inhibition of the immunosuppressive activity of TGF-beta, the main endogenous immunosuppression factor on the anticancer immunity.

\section{IL-13}

IL-13 is mainly produced by TH2 lymphocytes and basophils, and its effects are like to those exerted by IL$4^{24,25}$.

\section{IL-14}

IL-14 is one of the main B cell growth factors (BCGF). Then, it could play a stimulatory effect on the proliferation of hematologic malignancies ${ }^{33}$.

\section{IL-15}

IL-15 exerts immunological effects comparable to those of IL-2, with stimulation of T, B and NK cell proliferation ${ }^{15}$. In addition, IL-15 may induce effector memory cytotoxic $\mathrm{T}$ lymphocytes ${ }^{15}$. Preliminary clinical results would suggest that IL-15 may represent a new strategy in the immunotherapy of cancer, with results comparable to those exerted by IL-2, but with less side-effects ${ }^{15}$. There are two isoforms of IL-15, which is mainly produced by monocytes, macrophages and dendritic cells, even though it may be produced by several tissues.

\section{IL-16}

IL-16 is a still less investigated cytokine, characterized by several and often opposite functions, and provided by inflammatory effects due to a stimulation of the monocytemacrophage system ${ }^{34}$. Then, it is involved as a mediator of the inflammatory processes. In addition, it has also been proven to inhibit HIV replication ${ }^{34}$. 


\section{IL-17}

IL-17 is produced by TH17 lymphocytes, and it seems to constitute the main cytokine responsible for the onset of autoimmune mechanisms, since its main activity would consist of the inhibition of $\mathrm{T}$ reg cells, with a consequent diminished inhibitory control on the activation of autoreactive T lymphocytes ${ }^{17}$. The control of IL-17 is very complex, since it has appeared to be under a stimulatory mechanism played by IL- 1 beta and IL-6, while it is namely inhibited by IL- $12^{10}$, as well as by IL- $2^{5,35}$. More complex is the interaction between IL-17 and TGF-beta, since IL-17 inhibits TGF-beta secretion from $\mathrm{T}$ reg cells ${ }^{17}$, while the effects of TGF-beta on IL-17 secretion are depending on the concentrations of some other cytokines. In more detail, TGF-beta alone would play a major inhibitory action on IL-17 secretion, while it exerts a stimulatory role in the presence of IL- $6^{36}$ or IL- $23^{37}$. Therefore, IL-23 would also be involved as well as IL-17 in the pathogenesis of the autoimmune diseases ${ }^{38}$. As far as the possible influence of IL-17 on cancer is concerned, the inhibitory effect of IL-17 on the immunosuppressive action of $\mathrm{T}$ reg cells could be a potentially therapeutic effect in cancer, but it would be abrogated by the concomitant direct stimulatory action of IL-17 on cancer cell proliferation ${ }^{39}$. There are six isoforms of IL-17 (A, B, C, D, E, F), and the most biologically active form is IL-17A.

\section{IL-18}

IL-18 is a pleiotropic cytokine produced by several cells, including macrophages, hematopoietic cells and endothelial cells. It is involved in the regulation of innate and acquired immunity, as well as IL-12, even though with different effects ${ }^{9,40}$. In more detail, as well as IL-12 and IL15 , IL-18 is a potent inducer of gamma-IFN production by both NK cells and TH1 lymphocytes, and it would be involved in the pathogenesis of the autoimmune diseases ${ }^{41}$. In fact, the autoimmune diseases are often characterized by a concomitant increase in both IL-18 and gammaIFN blood concentrations. On the contrary, the allergic pathologies tend to be characterized by high levels of IL18 in association with low levels of gamma-IFN ${ }^{9,41}$. IL-18 would also exert an angiogenic action. Finally, IL-18 has also been proven to stimulate the appetite and to play a role in the development of obesity ${ }^{41}$.

\section{IL-19}

IL-19 is a member of IL-10 family, as well as IL-20, IL-22, and IL-24 $4^{42}$. IL-19 is namely produced by TH2 lymphocytes, as well as IL-20, IL-22, IL-24. High levels of IL-19, IL-20, and IL-24 have been described in autoimmunity, but it remains to be established whether they may play an inflammatory, or an anti-inflammatory action to counteract autoimmunity-related inflammatory status, such as IL-10.
Despite the controversial data of the literature, IL-19 would play a prevalent anti-inflammatory action. Moreover, IL19 has been shown to play an atheroprotective role ${ }^{43}$. In contrast, IL-20 and IL-24 would exert a predominant inflammatory activity.

\section{IL-20}

IL-20, as well as IL-19 and IL-24, would be involved in the pathogenesis of the autoimmune diseases by stimulating the release of inflammatory cytokines, even though it is still unclear whether it may be one of their causes, or the simple reaction to counteract their progression ${ }^{42.44}$.

\section{IL-21}

IL-21, which is produced by TH1 lymphocytes, has appeared to be a potentially effective antitumor cytokine, as well as IL-12. The antitumor activity of IL-21 seems to be due to its inhibitory action on T reg cells and on TH17 cell differentiation. Moreover, IL-21 in association with IL-12 may display an inhibitory effect on T reg and TH17 lymphocytes superior to that observed with IL-21 alone and IL-12 alone $\mathrm{e}^{45,46}$. IL-21 has also appeared to abrogate the increase in T reg cells induced by IL-2 plus TGF-beta ${ }^{47}$. Then, IL-21 could be effective in the immunotherapy of cancer in association either with IL-12, or IL-2.

\section{IL-22}

IL-22 is produced by several types of lymphocytes, including TH1, TH17 and NK cells ${ }^{48}$. IL-22 plays a proinflammatory effect by acting synergistically with TNFalpha, IL-1 beta and IL-17, but also by determining some unique effects through a direct stimulation of acute phase reactant production.

\section{IL-23}

IL-23 may be secreted by several lymphocytes, and its main effect is constituted by the stimulation of TH17 activation and IL-17 production, either alone, or in association with IL- ${ }^{49}$. Then, IL-23 would play an important role in the development of the autoimmune diseases ${ }^{50}$.

\section{IL-24}

IL-24 is produced by TH2 cells and monocytes. It has appeared to stimulate TNF-alpha and IL- 6 secretion. Then, it is involved in determining the inflammatory processes ${ }^{51}$.

\section{IL-25}

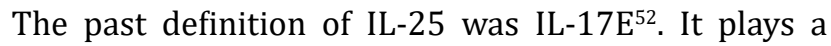
unique function within IL-17 cytokine family. IL-25 is produced by several cells, including TH2 lymphocytes, mast cells, and macrophages. It stimulates TH2 functions, with a consequent enhanced production of IL-4, IL-5, and IL-13. Moreover, IL-25 has also been proven to stimulate IL- 
17 secretion from TH17 lymphocytes. Then, it would play a role in the onset of allergic inflammation, including asthma. In addition, it would be also involved in the pathogenesis of the autoimmune diseases ${ }^{52}$.

\section{IL-26}

IL-26 is produced by both epithelial and immune cells, including TH1, TH17, and NK cells. It may exert antiviral and antimicrobial effects. It would be also involved in the chronicity of inflammation ${ }^{53}$.

\section{IL-27}

The main source of IL-27 is represented by the antigen presenting cells, then the dendritic cells and the activated macrophages ${ }^{54}$. IL-27 was initially thought to be a proinflammatory cytokine, but now it is known that it may play an immunoregulatory role with anti-inflammatory activity by inhibiting IL-17A and IL-2 secretion ${ }^{54}$. Moreover, it has also been proven to stimulate IL-10 secretion. Then, it could display a therapeutic role in the autoimmunity, but not of cancer, because of IL-10-induced immunosuppression.

\section{IL-28}

IL-28, produced by dendritic cells, TH17, and virusinfected cells, has been proven to play an anti-viral activity by inhibiting viral replication ${ }^{55}$.

\section{IL-29}

IL-29 is mainly produced by dendritic cells, monocytes and TH17 lymphocytes. As well as IL-28, it is a cytokine with IFN-like activity ${ }^{56}$. Then, it may inhibit viral replication with mechanisms like to those of IFN-alpha. It exerts an antiinflammatory effect in the allergic diseases. In contrast, its role in cancer is still controversial, since either pro-tumoral and anti-tumoral activities have been described.

\section{IL-30}

IL-30, represented by the p28 subunit of IL-27, displays an anti-inflammatory activity like to IL-27 by inhibiting IL-17A production ${ }^{57}$. Moreover, it has been recently demonstrated that IL-30 expression may predict a negative prognosis at least in some tumor histotypes, including breast and prostate tumors ${ }^{1,58}$.

\section{IL-31}

IL-31 is a member of IL-6 family, then it is a 4-helix bundle cytokine ${ }^{1}$. IL-31 is produced by TH2 lymphocytes, as well as by eosinophils, and it acts on IL-31 receptor A and oncostatin $\mathrm{M}$ receptor. IL-31 secretion correlates with that of two other TH2 cytokines, IL-4 and IL-13, and it seems to be involved in atopic dermatitis. Moreover, IL-31 correlates with the intensity of pruritus ${ }^{59}$.

\section{IL-32}

IL-32 may be produced by several peripheral blood mononuclear cells (PBMCs), and there are various isoforms of IL-32, probably provided by different functions ${ }^{1}$. IL32 stimulates the production of several inflammatory cytokines, including TNF-alpha, IL-1 beta, IL-6, and several chemokines, then it plays an important inflammatory activity and would be involved in several autoimmune diseases ${ }^{60}$, as well as in cancer progression ${ }^{1}$. Therefore, IL32 may be considered as an inflammatory and pro-tumoral cytokine.

\section{IL-33}

IL-33 is a member of IL-1 family, and it is produced by TH2 cells, eosinophils, basophils and mast cells. IL-35 stimulates IL-4, IL-5 and IL-13 secretion, and it may allow eosinophilia and an increased secretion of $\operatorname{IgE}$, then it is involved in the allergic disorders ${ }^{61}$.

\section{IL-34}

IL-34 is produced by macrophage and $\mathrm{T}$ reg cell systems, by keratinocytes in the skin and neurons in the brain. IL-34 has been proven to interact with monocytecolony stimulation factor (M-CSF) in several functions, including the promotion of the osteoblastogenesis ${ }^{62}$, then it could deserve a potential therapeutic activity in osteoporosis. On the contrary, IL-11 has appeared to stimulate the osteoclastogenesis ${ }^{63}$. Then, bone metabolism would also be under a cytokine regulation, and IL-34 and IL-11 would constitute a functional axis with opposite effects. In addition, IL-34 may stimulate T reg cell system ${ }^{64}$, with a consequent induction of an immunosuppressive status, which may prevent acute rejection after organ transplantation, but on the same time it may promote tumor progression. Finally, IL-34 may act as a profibrotic factor, then it would be involved in age-related organ fibrosis, as well as TGF-beta ${ }^{65}$.

\section{IL-35}

IL-35 was discovered since 1997, but not named IL-35 until $2017^{1}$. IL-35 is mainly produced by $\mathrm{T}$ reg cells and regulatory $B$ lymphocytes, and it exerts a strong suppressive activity on several immune functions by activating $\mathrm{T}$ reg cell system, with a consequent enhanced production of both TGF-beta and IL-10, and a following inhibition of TH1 cells and IL-2 production ${ }^{66}$. Moreover, IL-35 has also been shown to inhibit TH17 cell proliferation ${ }^{66}$, then it could be more effective than TGF-beta and IL-10 themselves in the treatment of autoimmune disease. On the contrary, IL-35induced stimulation of $\mathrm{T}$ reg cell system might negatively influence the prognosis of human tumors. 


\section{IL-36}

IL-36 is a proinflammatory cytokine produced by naïve CD4 T cells, skin keratinocytes, myeloid cells, Langerhans cells, and mucosal epithelium. Its proinflammatory role is due to a stimulating effect on the secretion of most inflammatory cytokines, including TNF-alpha, IL-6, IL-17A and IL-23 ${ }^{67}$. However, in contrast to the behaviour of other inflammatory cytokines, which play a major inhibitory role on IL-2 secretion, IL-36, as well as IL-12, may also promote TH1 differentiation ${ }^{67}$. In any case, IL-36 would play an essential role in skin defense and tissue repair in the gut ${ }^{1}$.

\section{IL-37}

IL-37 is also an important cytokine of the IL-1 family, with structural similarities with IL-18 (1), which is present in five isoforms (IL-37 a, b, c, d, e), but it is paradoxically provided by an anti-inflammatory activity, since it has been proven to completely suppress the secretion of the main pro-inflammatory cytokines, including TNF-alpha, IL-6, and IL- 1 beta, as well as several chemokines ${ }^{68}$. Then, IL-37 is an important cytokine in immune homeostasis as an immune regulator, with potential therapeutic benefits in the immunotherapy of cancer by blocking the immunosuppressive action of IL-6, IL-1 beta and TNF-alpha, as well as of autoimmune pathologies by counteracting inflammatory cytokine-induced tissue damage.

\section{IL-38}

IL-38 is another member of IL-1 family, produced by several cell types, including skin, thymus, tonsil, liver and brain, and provided by a typical pro-inflammatory activity by stimulating the secretion of several other proinflammatory cytokines, including IL-1 beta, IL-22, IL-17A and IL-36 itself ${ }^{69}$.

\section{IL-39}

IL-39 is the most recently discovered member of IL-12 family ${ }^{70}$. It is mainly secreted by activated B lymphocytes, dendritic cells and macrophages. It mediates the inflammatory response by promoting the differentiation of neutrophils and their chemotactic ability.

\section{IL-40}

IL-40 is the last discovered interleukin ${ }^{1}$, produced by activated B cells, bone marrow cells and some tissues, such as the mammary gland. It plays an important role in normal B cell function, particularly for IgA secretion at local level. The action of IL-40 is enhanced by TGF-beta ${ }^{1}$, as well as previously observed for other stimulating factors for B lymphocytes ${ }^{71}$.

\section{The similarity among the Different Interleukins}

According to the biological behaviour existing in Nature, several biological functions are present in a double similar forms to compensate possible deficiencies of the single form. Then, even though in a schematic way, the 40 interleukins could be subdivided in 20 fundamental couples characterized by similar and complementary functions. As shown in Table 2, it is possible to identify

Table 2. The 20 functional couples of the 40 human interleukins.

\begin{tabular}{|c|c|}
\hline COUPLE OF INTERLEUKINS & SIMILAR FUNCTIONS \\
\hline IL-1 - IL-18 * & Induction of the acute inflammatory response \\
\hline IL-2 - IL-12*+ & Stimulation of antigen-independent and- dependent anticancer immunity \\
\hline IL-3 - IL-7 + & Development of hematopoietic lymphocyte and monocyte systems \\
\hline IL-4 - IL-13 * & Stimulation of histamine release \\
\hline IL-5 - IL-25* & Stimulation of eosinophil generation and functions \\
\hline IL-6 - IL-22* & Activation of the acute inflammatory reaction \\
\hline IL-8 - IL-39 * & Activation of neutrophil functions \\
\hline IL-9-IL-32*+ & Predisposition to autoimmunity and cancer \\
\hline IL-10 - IL-35 + & Immunosuppression of IL-2-dependent immunity, including the anticancer one \\
\hline IL-11- IL-34 + & Regulation of bone metabolism \\
\hline IL-15-IL-21*+ & Cooperation with IL-2 and IL-12 in the induction of anticancer immunity \\
\hline IL-14 - IL-40 * & Stimulation of B lymphocytes \\
\hline IL-16 -IL-26 * & Anti-viral activity \\
\hline IL-17 - IL-23 * & Activation of TH17 system in inhibiting T reg cell functions \\
\hline IL-19-IL-37+ & Stimulation of TGF-beta production and inhibition of inflammatory cytokines \\
\hline IL-20 - IL-24* & Stimulation of inflammatory cytokine TNF-alpha and IL- 6 production \\
\hline IL-27 - IL-30 + & Anti-inflammatory action by inhibiting IL-17 cell system \\
\hline IL-28 - IL-29 * & Anti-viral activity \\
\hline IL-31 - IL-33 * & Stimulation of IL-4 and IL-13 secretion \\
\hline IL-36 - IL-38 * & Stimulation of IL- 6 and TNF-alpha secretion \\
\hline
\end{tabular}


Table 3. Main biological effects of the 40 interleukins and their site of production.

\begin{tabular}{|c|c|c|}
\hline INTERLEUKIN & SITES OF ORIGIN & MAIN IMMUNOBIOLOGICAL FUNCTIONS \\
\hline IL-1 beta & Macrophages & Stimulation of IL- 6 secretion, fever and interactions with IL-18 \\
\hline IL-2 & TH1 cells & Lymphocytosis, eosinophilia, LAK cell generation \\
\hline IL-3 & Macrophages, stromal cells & Multi-colony stimulating factor \\
\hline IL-4 & TH2 cells, basophils & Stimulation of histamine release \\
\hline IL-5 & $\mathrm{TH} 2$ & Stimulation of eosinophil generation \\
\hline IL-6 & Macrophages & Stimulation of CRP hepatic production, severe hypotension \\
\hline IL-7 & Thymic stromal cells & T lymphocyte development and differentiation \\
\hline IL-8 & Macrophages, stromal cells & Like IFN-activity \\
\hline IL-9 & TH2 cells & Stimulation of T reg and TH17 lymphocytes \\
\hline IL-10 & $\mathrm{TH} 2$ cells, macrophages & Inhibition of IL-2 and IL-12 secretions \\
\hline IL-11 & Stromal cells & Stimulation of platelet generation, osteoclast cell stimulation \\
\hline IL-12 & Dendritic cells & Stimulation of TH1 cells, inhibition of T reg and TH17 cells \\
\hline IL-13 & TH2 cells & IL-4-like activity \\
\hline IL-14 & Macrophages & Growth factor for B lymphocytes \\
\hline IL-15 & Dendritic cells, macrophages & Stimulation of T cells, B cells and NK cells \\
\hline IL-16 & Macrophages, stromal cells & Macrophage stimulation, inhibition of HIV replication \\
\hline IL-17 & TH17 lymphocytes & Inhibition of T reg cells, reciprocal stimulation with IL-6 \\
\hline IL-18 & Macrophages, endothelium & Inflammatory activity in cooperation with IL-1 beta \\
\hline IL-19 & TH2 lymphocytes & Anti-atherosclerotic activity \\
\hline IL-20 & Activated T lymphocytes & Stimulation of inflammatory cytokine secretion \\
\hline IL-21 & TH1 lymphocytes & Inhibition of T reg cells secretion and TH17 differentiation \\
\hline IL-22 & TH1, TH17, NK lymphocytes & Stimulation of TNF, IL-1, IL-17 and CRP production \\
\hline IL-23 & Several lymphocyte types & Stimulation of IL-17 secretion \\
\hline IL-24 & TH2 cells, monocytes & Stimulation of TNF-alpha and IL-6 secretion \\
\hline IL-25 & $\mathrm{TH} 2$, monocytes, mast cells & Stimulation of IL-4, IL-5, IL-13 and IL-17 secretion \\
\hline IL-26 & TH1, TH17, NK, epithelial cells & Antiviral and antimicrobial activity \\
\hline IL-27 & Dendritic cells, macrophages & Inhibition of IL-17 and IL-2 secretion \\
\hline IL-28 & Dendritic cells, TH17 cells & Antiviral activity \\
\hline IL-29 & Dendritic cells, TH17 cells & IFN-like activity \\
\hline IL-30 & Several immune cells & Inhibition of IL-17 secretion \\
\hline IL-31 & TH2 cells, eosinophils & Stimulation of IL-4 and IL-13 secretion \\
\hline IL-32 & PBMC & Stimulation of TNF-alpha, IL- 6 and IL-1 beta secretion \\
\hline IL-33 & $\mathrm{TH} 2$, basophils, eosinophils & Eosinophilia and increased IgE production \\
\hline IL-34 & T reg cells, macrophages & Interaction with M-CSF, osteoblastogenetic activity \\
\hline IL-35 & T reg cells, B reg lymphocytes & Stimulation of T reg cell activity, inhibition of IL-2 secretion \\
\hline IL-36 & Naïve CD4+cells, keratinocytes & Stimulation of TNF-alpha, IL-6, IL-17 and IL-23 secretion \\
\hline IL-37 & Several immune cells & Anti-inflammatory role inhibiting TNF, IL-6 and IL-1 release \\
\hline IL-38 & Several cell types & Stimulation of IL-1 beta, IL-22 and IL-17 secretion \\
\hline IL-39 & B lymphocytes, dendritic cells & Stimulation of neutrophil differentiation and chemotaxis \\
\hline IL-40 & B cells and several tissue cells & Stimulation of B lymphocytes \\
\hline
\end{tabular}

* Inflammatory ILs: IL-1, IL-6, IL-8, IL-17, IL-18, IL-20, IL-22, IL-23, IL-24, IL-25, IL-32, IL-36, IL-38

* Anti-inflammatory ILs: IL-10, IL-35, IL-37 (plus TGF-beta)

Dual effects: IL-2, IL-12, IL-3, IL-4, IL-5, IL-11, IL-13

+ Antitumor ILs: clinically proven antitumor ILs: IL-2, IL-12, IL-15; possible antitumor ILs: IL-7, IL-21, IL-37

+ ProtumorILs: IL-1, IL-6,IL-8, IL-10,IL-14,IL-17, IL-18,IL-20,IL-22, IL-23, IL-24,IL-25, IL-32, IL-35, IL-36, IL-38 (plus TGF-beta)

12 pro-inflammatory couples of interleukins $(\mathrm{n}=24)$, 5 anti-inflammatory couples of interleukins $(n=10)$, and 3 couples of interleukins with dual pro- and antiinflammatory activities $(\mathrm{n}=6)$. Table 3 shows the principal immunobiological effects of each single interleukin, its major source of origin, and its main proven interactions with the other cytokines. It clearly appears that the dynamics involved in regulating cytokine secretions is beyond the simple mechanic logical reasoning, and it is more similar to the symbolic imagination of the Tree of Life, the so-called Sephirot of the cabalistic tradition ${ }^{72}$, which could be useful for meditation and for the identification of possible analogic connection occurring among the different cytokine secretion. 


\section{Conclusions}

If clinically we exclude the investigation of the cytokine network, the human physiology cannot be considered complete. The aim of the present review is to elaborate a preliminary fundamental physiology of the cytokine network and no pathogenesis of the various systemic disease may be considered as definitely understood. On the contrary, the complete knowledge of the physiology of the cytokine network will allow the generation of a new future medical Science, which perhaps will realize a complete resolution of all human pathologies.

\section{References}

1. Catalan-Dibene J, McIntyre LL, Zlotnik A. Interleukin 30 to interleukin 40. J Interferon Cytokine Res. 2018; 38: 423-439.

2. Rubinow DR. Brain, behaviour and immunity: an interactive system. J Natl Cancer Inst Monogr. 1987; 10: 79-82.

3. Mantovani A, Allavena P, Sica A, et al. Cancer-relatedinflammation. Nature. 2008; 454: 436-444.

4. Korn T, Bettelli E, Oukka M, et al. IL-17 and Th17 cells. Annu Rev Immunol. 2009; 27: 485-517.

5. Lissoni P. Therapy implications of the role of intereluin-2 in cancer. Expert Rev Clin Immunol. 2017; 13: 491-498.

6. Elkassar N, Gress RE. An overview of IL-17 biology and its use in immunotherapy. J Immunotoxicol. 2010; 1-7.

7. Towne JE, Renshaw BR, Douangapanya J, et al. Interleukin-36 (IL-36) ligands require processing for full agonist (IL-36 alpha, IL-36 beta, and IL-36 gamma) or antagonist (IL-36Ra) activity. J Biol Chem. 2011; 286: 42594-42602.

8. Boraschi D, Lucchesi D, Hainzl S, et al. IL-37: a new anti-inflammatory cytokine of the IL-1 family. Eur Cytokine Netw. 2011; 22: 127-147.

9. Dinarello CA, Kaplanski G. Interleukin-18 treatment options for inflammatory diseases. Expert Rev Clin Immunol. 2005; 1: 619-632.

10. Murphy CA, Langrish CL, Chen Y, et al. Divergent pro- and antiinflammatory role for IL-23 and IL-12 in joint autoimmune inflammation. J Exp Med. 2003; 198: 1951-1957.

11. Lissoni $P$, Barni S, Tisi E, et al. In vivo biological results of the association between interleukin-2 and interleukin-3 in the immunotherapy of cancer. Eur J Cancer. 1993; 29A: 1127-1132.

12. Walmsley M, Butler DM, Marinova-Mutafchieva L, et al. An antiinflammatory role for interleukin-11 in established murine collageinduced arthritis. Immunology. 1998; 95: 31-37.

13. Cavaillon JM. Pro- versus anti-inflammatory cytokines: myth or reality. Cell Med Biol. 2001; 47: 695-702.

14. Elkassar N, Gress RE. An overview of IL-7 biology and its use in immunotherapy. J Immunotoxicol. 2010; 7: 1-7.

15. Waldmann TA. Interleukin-15 in the treatment of cancer. Expert Rev Clin Immunol. 2014; 10: 1689-1701.

16. Gollob JA, Veenstra KG, Parker RA, et al. Phase I trial of concurrent twice-weekly recombinant human interleukin-12 plus low-dose IL-2 in patients with melanoma or renal cell carcinoma. J Clin Oncol. 2003; 21: 2564-2573.

17. Lissoni $\mathrm{P}$, Barni $\mathrm{S}$, Mandalà $\mathrm{M}$, et al. Immunobiological effects of interleukin-2, interleukin-12 or interleukin-2 plus interleukin-12 in metastatic cancer patients. Int J Immunotherapy XIV. 1998; 41-44.

18. Dennis KL, Blatner NR, Gounari F, et al. Current status of IL-10 and regulatory T cells in cancer. CurrOpin Oncol. 2013; 25: 637-645.
19. Dinarello CA. Interleukin 1 and interleukin 18 as mediators of inflammation and the aging process. Am J Clin Nutr. 2006; 83: 447S-455S.

20. Banks RE, Patel PM, Selby PJ. Interleukin-12: a new clinical player in cytokine therapy. Br H Cancer. 1995; 71: 655-659.

21. Laurence $\mathrm{A}$, Tato $\mathrm{CM}$, Davidson $\mathrm{TS}$, et al. Interleukin-2 signaling via STAT5 constrains T helper 17 cell generation. Immunity. 2007; 26: 371-381.

22. He J, Zhang X, Li Z. Low-dose interleukin-2 treatment selectively modulates CD4+ $\mathrm{T}$ cells subsets in patients with systemic lupus erythematosus. Nat med. 2016; 22: 991-993.

23. Dougan M, Dranoff G, Dougan SK. GM-CSF, IL-3, and IL-5 family of cytokines: regulators of inflammation. Immunity. 2019; 50: 798-811.

24. Redrup AC, Howard BP, MacGlashan DW Jr, et al. Differential regulation of IL-4 and IL-13 secretion by human basophils: their relationship to histamine release in mixed leukocyte cultures. J Immunol. 1998; 160: 1957-1964.

25. Andoh A, Hata K, Araki Y. Interleukin (IL)-4 and IL-17 synergistically stimulate IL-6 secretion in human colonic myofibroblasts. Int J Mol Med. 2002; 10: 631-634.

26. Damas $P$, Ledoux D, Nys $M$, et al. Cytokine serum levels during severe sepsis in human IL-6 as a marker of severity. Ann Surg. 1992; 215: 356-362.

27. Jiang Q, Li WQ, Aiello FB, et al. Cell biology of IL-7, a key lymphotrophin. Cytokine Growth Factor Rev. 2005; 16: 513-533.

28. Kasahara T, Mukaidu N, Yamashita K, et al. IL-1 and TNF-alpha induction of IL-8 and monocyte chemotactic activating factor (MCAF) mRNA expression in a brain astrocytoma cell line. Immunology. 1991; 74: 60-67.

29. Fischer M, Bijman M, Molin D, et al. Increased serum levels of interleukin-9 correlate to negative prognostic factors in Hodgkin's lymphoma. Leukemia. 2003; 17: 2513-2516.

30. Elyaman W, BradshaWem, Uyttenhove C, et al. IL-9 induces differentiation of TH17 cells and enhances function of FoxP3+ natural regulatory T cells. Proc Natl Acad Sci Usa. 2009; 106: 12885-12890.

31. Visekruna A, Ritter J, Scholz T, et al. Tc9 cells, a new subset of CD8+ T cells, support Th2-mediated airway inflammation. Eur J Immunol. 2013; 43: 606-618.

32. Goldman SJ. Preclinical biology of interleukin-11: a multifunctional hematopoietic cytokine with potential thrombopoietic activity. Stem Cells Dayt. 1995; 13: 462-471.

33. Ballaun C. Interleukin 14. In Handboock of Immunopharmacology. 1998; 217-219.

34. Mathy NL, Scheuer W, Lanzendorfer M, et al. Interleukin 16 stimulates the expression and production of proinflammatory cytokines by human monocytes. Immunology. 2000; 100: 63-69.

35. Kryczek L, Wei S, Vatan L, et al. Cutting edge: opposite effects of IL1 and IL- 2 on the regulation of IL-17+ T cell pool IL-1 subverts IL-2mediate suppression. J Immunol. 2007; 179: 1423-1426.

36. Chen Z, Tato CM, Muul L, et al. Distinct regulation of IL-17 in human helper T lymphocytes. Arthritis Rheum56. 2007; 2936-2946.

37. McKenzie BS, Kastelein RA, Cua DJ. Understanding the IL-23-IL-17 immune pathway. Trends Immunol. 2006; 27: 17-23.

38. Langrish CL, Chen Y, Blumenschein WM, et al. IL-23 drives a pathogenic T cell population that induces autoimmune inflammation. J Exp Med. 2005; 201: 233-240.

39. Wang L, Yi T, Kortylewski M, et al. IL-17 can promote tumor growth through an IL-6-Stat3 signaling pathway. J Exp Med. 2009; 206: 1457 1464 . 
40. Wawronsky S, Druszczynska M, Kowalewicz-Kulbat $M$, et al Interleukin-18 (IL-18) as a target for immune intervention. Acta Biochim Pol. 2016; 63: 59-63.

41. Dinarello CA. Interleukin-18 and the pathogenesis of inflammatory diseases. SeminNephrol. 2007; 27: 98-114.

42. Leng RX, Pan HF, Tao JH, et al. IL-19, IL-20 and IL-24: potential therapeutic targets for autoimmune diseases. Expert OpinTher Targets. 2011; 15: 119-126.

43. Jain S, Gabunia K, Kelemen SE, et al. The anti-inflammatory cytokine interleukin 19 is expressed in and angiogenic for human endothelial cells. ArterosclerThrombVascBiol. 2010; 31: 167-175.

44. Wei CC, Hsu YH, Li HH, et al. IL-20: biological functions and clinical implications. J Biomed Sci. 2006; 13: 601-602.

45. Peluso I, Fantini MC, Fina D, et al. IL-21 counteracts the regulatory T cell-mediated suppression of human CD4+ T lymphocytes. J Immunol. 2007; 178: 732-739.

46. Tian Y, Yuan C, Ma D, et al. IL-21 and IL-12 inhibit differentiation of $\mathrm{T}$ reg and TH17 cells and enhance cytotoxicity of peripheral blood mononuclear cells in patients with cervicakl cancer. Int J Gynecol Cancer. 2011; 21: 1672-1678.

47. Battaglia A, Buzzonetti A, Baranello C, et al. Interleukin-21 (IL-21) synergizes with IL-2 to enhance T cell receptor-induced human T cell proliferation and counteracts IL-2/transforming growth factor-betainduced regulatory T cell development. Immunology. 2013; 139: 109120.

48. Wolk K, Witte E, Witte $\mathrm{K}$, et al. Biology of interleukin-22. SeminImmunopathol. 2010; 32: 17-31.

49. Zenewicz LA, Flavell RA. Recent advances in IL-22 biology. Int Immunol. 2011; 23: 159-163.

50. Tang $\mathrm{C}$, Chen S, Qion $\mathrm{H}$, et al. Interleukin-23 as a drug target for autoimmune inflammatory diseases. Immunology. 2012; 135: 112 124.

51. Wang M, Liang P. Interleuin-24 and its receptors. Immunology. 2005; 114: $166-170$.

52. Valizadeh A, Khosravi A, Zadeh LJ, et al. Role of IL-25 in immunity. J Clin Diagn Res. 2015; 9: 1-4.

53. Itoh T, Hatano R, Komiya E, et al. Biological effects of IL-26 on T cellmediated skin inflammation, including psoriasis. J Invest Dermatol. 2019; 139: 878-889.

54. Fitzgerald DC, Zhang GX, El-Behi M, et al. Suppression of autoimmune inflammation of the central nervous system by interleukin 10 secreted by interleukin 27-stimulated T cells. Nat Immunol. 2007; 8: 1372-1379.

55. Wolik K, Witte K, Sabat R. Interleukin-28 and interleukin-29: novel regulators of skin biology. J Interferon Cytokine Res. 2010; 30: 617628.
56. Kelm NE, Zhu Z, Ding VA, et al. The role of IL-29 in immunity and cancer. Critical Rev and Hematol. 2016; 106: 91-98.

57. Stumhofer IS, Laurence A, Wilson EH, et al. Interleukin-27 negatively regulates the development of interleukin 17-producing $\mathrm{T}$ helper cells during chronic inflammation of the central nervous system. Nat Imunol. 2006; 7: 937-945.

58. Sorrentino C, Ciummo SL, Cipollone G, et al. Interleukin-30/IL27p28 shapes prostate cancer stem-like cell behavior and its critical role for tumor onset and metastatization. Cancer Res. 2018; 78: 2654-2668.

59. Neis MM, Peters B, Dreuw A, et al. Enhanced expression levels of IL-31 correlate with IL-4 and IL-13 in atopic and allergic contact dermatitis. J Allergy Clin Immunol. 2006; 118: 930-937.

60. Felaco P, Castellani ML, De Lutiis MA, et al. IL-32: a newly-discoveredpro inflammatorycytokine. J BiolRegulHomeost Agents. 2009; 23: 141-147.

61. Yagami A, Orihara K, Morita H, et al. IL-33 mediates inflammatory response in human lung tissue cells.J Immunol. 2010; 185: 5743-5750.

62. Chen Z, Buki K, Vaaramieni J, et al. The critical role of IL-34 in osteoclastogenesis. PLoS. 2011; 6(4): e18689.

63. Jimi E, Shuto T, Ikebe T, et al. Basic fibroblast growth factor inhibits osteoclast-like formation. J Cell Physiol. 1996; 168: 395-402.

64. Bezie S, PIcarda E, Ossart J, et al. IL-34 is a T reg-specific cytokine and mediates transplant tolerance. J Clin Invest. 2015; 125: 3952-3964.

65. Galligan CL, Fish EN. Interleukin-34 promotes fibrocyte proliferation. I Interferon Cytokine Res. 2017; 37: 440-448.

66. Wirtz S, Billmeier U, McHedlidze T, et al. Interleukin-35 mediates mucosal immune responses that protect against T-cell-dependent colitis. Gastroenterology. 2011; 141: 1875-1886.

67. Towne JE, Renshaw BR, Douangpanya J, et al. Interleukin-36 (IL-36) ligands require processing for full agonist (IL-36 alpha, IL-36 beta, and IL-36 gamma) or antagonist (IL-36Ra) activity. J Biol Chem. 2011; 286: 42594-42602.

68. Nold MF, Nold-Petry CA, Zepp JA, et al. IL-37 is a fundamental inhibitor of innate immunity. Nat Immunol. 2010; 11: 104-1022.

69. Rudloff I, Godsell J, Nold-Petry CA, et al. Brief report: interleukin-38 exerts anti-inflammatory functions and it is associated with disease activity in systemic lupus erythematosus. Arthritis Rheumatol. 2015; 67: 3219-3225

70. Wang X, Wei Y, Xiao H, et al. A novel 23p19/Ebi3 (IL-39) cytokine mediates inflammation in lupus-like mice. Eur J Immunol. 2016; 46: 1343-1350.

71. Sonoda E, Matsumoto R, Hitoshi Y, et al. Transforming growth factorbeta induces IgA production and acts additively with interleukin 5 for IgA production. J Exp Med. 1989; 170: 1415-1420.

72. Abramo G, Crivelli N. Cabalistic comment to "Shir ha-Shirim". ebook (ed) Rome Italy 1995. 